\title{
Progress on four flavor QCD with the HISQ action
}

\section{The MILC Collaboration:}

\section{A. Bazavov ${ }^{*}{ }^{a}$ C. Bernard, ${ }^{b}$ C. DeTar, ${ }^{c}$ W. Freeman, ${ }^{a}$ Steven Gottlieb, ${ }^{d}$ U.M. Heller,${ }^{e}$ J.E. Hetrick, ${ }^{f}$ J. Laiho, ${ }^{b}$ L. Levkova, ${ }^{c}$ J. Osborn, ${ }^{g}$ R. Sugar, ${ }^{h}$ D. Toussaint,${ }^{a}$ and} R.S. Van de Water ${ }^{i}$

${ }^{a}$ Department of Physics, University of Arizona

Tucson, AZ 85721, USA

E-mail: bazavovephysics.arizona.edu

${ }^{b}$ Department of Physics, Washington University

St. Louis, MO 63130, USA

${ }^{c}$ Physics Department, University of Utah

Salt Lake City, UT 84112, USA

${ }^{d}$ Department of Physics, Indiana University

Bloomington, IN 47405, USA

${ }^{e}$ American Physical Society

One Research Road, Box 9000, Ridge, NY 11961, USA

${ }^{f}$ Physics Department, University of the Pacific Stockton, CA 95211, USA

${ }^{g}$ Argonne Leadership Computing Facility, Argonne National Laboratory

Argonne, IL 60439, USA

${ }^{h}$ Department of Physics, University of California

Santa Barbara, CA 93106, USA

${ }^{i}$ Department of Physics, Brookhaven National Laboratory

Upton, NY 11973, USA

We describe recent progress on generation of gauge configurations using the Highly Improved Staggered Quark (HISQ) action that was designed by the HPQCD/UKQCD collaboration. The HISQ action requires two levels of smearing with a reunitarization of the links before the second smearing. We describe how we deal with the occurrence of occasional large forces arising from the reunitarization step. The MILC collaboration is currently generating ensembles with approximate lattice spacings of $0.15,0.12,0.09$, and $0.06 \mathrm{fm}$, with the strange and charm quark masses close to their physical values and the mass of the light quarks $m_{l}$ set to $0.2 m_{s}$. We present recent results for pion taste splittings, light hadron masses, the static potential, the $\eta_{c}$ dispersion relation and the topological susceptibility.

The XXVII International Symposium on Lattice Field Theory - LAT2009

July 26-31 2009

Peking University, Beijing, China

\footnotetext{
${ }^{*}$ Speaker.
} 


\section{Introduction}

The MILC collaboration has been using the asqtad action [1] for more than a decade for its generation of $2+1$ flavor dynamical quark configurations. Over forty ensembles covering six lattice spacings from $a \approx 0.18 \mathrm{fm}$ to $0.045 \mathrm{fm}$ and a wide range of quark masses have enabled us to explore both the continuum and chiral limits [2].

Recently, the Highly Improved Staggered Quark (HISQ) [3] action was developed by the HPQCD/UKQCD collaboration. This action requires two levels of smearing with a reunitarization of the links before the second smearing. Although it is more expensive to simulate than the asqtad action, its reduced taste symmetry breaking makes the HISQ action an attractive possibility for future calculations. It also allows us to include a dynamical charm quark with reasonable accuracy at current lattice spacings. The goal of this initial work is to generate several ensembles with a fixed ratio of light to strange quark masses and to study the approach to the continuum limit.

Large forces arise during molecular dynamics updating when the determinant of a link at the first level of smearing is close to vanishing [ [ $\bigoplus$. This problem is dealt with by a slight change in the guiding action, the effect of which can be eliminated via the accept/reject step at the end of each trajectory.

The MILC collaboration is currently generating ensembles with lattice spacings of about 0.15 , $0.12,0.09$, and $0.06 \mathrm{fm}$, with the strange and charm quark masses near their physical values and the mass of the light quarks $m_{l}$ set to $0.2 m_{s}$. In the future, we plan to generate additional ensembles with lighter up and down quark masses. We present recent results on pion taste splittings, light hadron masses, the $\eta_{c}$ dispersion relation and the topological susceptibility.

\section{Implementation of the HISQ action}

The HISQ action was developed to go beyond the asqtad action in its improvement of tastesymmetry. Links in the Dirac operator are replaced by

$$
U^{\prime}=\mathscr{F}_{2} \mathscr{U} \mathscr{F}_{1} U
$$

where smearing level 1 denoted $\mathscr{F}_{1}$ is Fat 7 ; reunitarization is denoted by $\mathscr{U}$; and smearing level 2 denoted $\mathscr{F}_{2}$ is asq smearing.

To calculate the fermion force, we must take the derivative of the action with respect to the gauge link. With multilevel smearing, we must use the chain rule [5], 6]. For the fermion force, we have:

$$
\frac{\partial S_{f}}{\partial U}=\frac{\partial S_{f}}{\partial X} \frac{\partial X}{\partial W} \frac{\partial W}{\partial V} \frac{\partial V}{\partial U},
$$

where $U$ are the fundamental gauge links, $V$ represent fat links after level 1 smearing, $W$ are the result of reunitarizing $V$, and $X$ are the links after asq smearing. Conveniently, code for three of the derivatives is the same as for the asqtad action (although the arguments may be different):

$$
\frac{\partial S_{f}}{\partial X}, \frac{\partial X}{\partial W}, \frac{\partial V}{\partial U}
$$

After the first level of smearing, the link $V$ must be projected into $U(3)$. This required some new code. We define: $W=V Q^{-1 / 2}$, with $Q=V^{\dagger} V$. The method to calculate the inverse square 


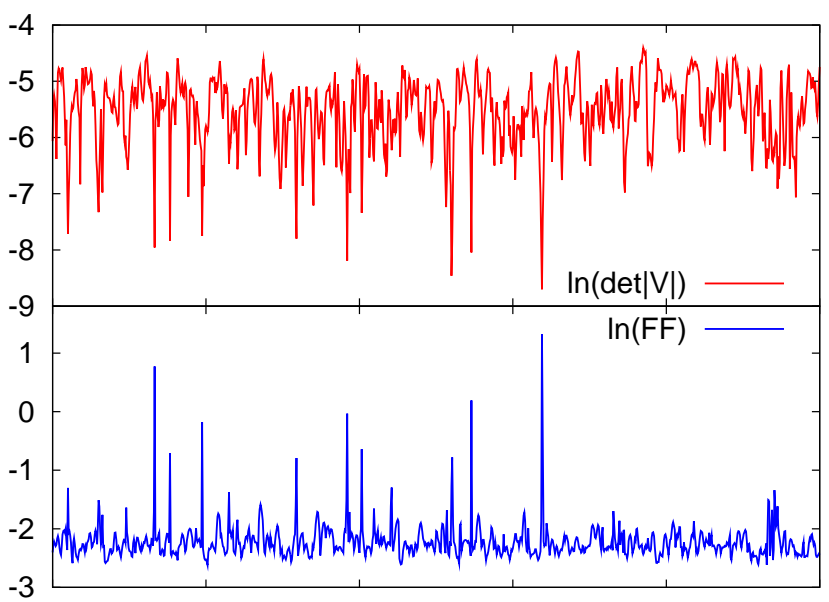

Figure 1: Time history of the minimum value of $\operatorname{det}|V|$ (upper plot) and the maximum magnitude of the fermion force (lower plot) 㺻. Note how the large spikes in the fermion force are correlated with near vanishing of $\operatorname{det}|V|$. The lattice spacing is $\approx 0.13 \mathrm{fm}$.

root, based on the Cayley-Hamilton theorem, had already been applied by Morningstar \& Peardon [7], and Hasenfratz, Hoffman \& Shaefer [8].

$$
Q^{-1 / 2}=f_{0}+f_{1} Q+f_{2} Q^{2} .
$$

We just need to find the eigenvalues of $Q$.

A significant issue in implementing molecular dynamics based on this algorithm is that there can be large changes in the action resulting from large forces during the integration of the equations of motion [ $₫$. In Fig. 1, we see that there is a correlation between the large forces and low values of $\operatorname{det}|V|$. In Fig. Q , we show the fermion force rescaled by $1 / \Delta t$ for runs with three different lattice spacings. (These two graphs are based on exploratory runs details of which can be found in Ref. [4].) It appears that as we approach the continuum, there are fewer large forces. As the fundamental links $U$ are varying more smoothly, we should not be surprised that $V$ is less likely to have a small determinant.

We implement an "eigenvalue filter" to tame the large forces. If the smallest eigenvalue of a smeared link $V$ is smaller than a certain cutoff, we make the replacement $Q^{-1 / 2} \rightarrow(Q+\delta I)^{-1 / 2}$, where $\delta$ is typically set to $5 \times 10^{-5}$. Although this modifies the guiding Hamiltonian, the integration algorithm is still reversible and area preserving. As long as we use the RHMC algorithm [9], the accept/reject step at the end of each MD trajectory ensures the correct equilibrium distribution.

\section{Testing the HISQ action}

For our initial test of the HISQ action, we are generating ensembles with $m_{l}=0.2 m_{s}$ and lattice spacings varying from $a \approx 0.15$ to $0.06 \mathrm{fm}$. Table 1 shows the masses, lattice dimensions and acceptance rate on our tuning runs. In Table 2, we consider the production runs at the two intermediate lattice spacings. This range of lattice spacings is comparable to what we have with 


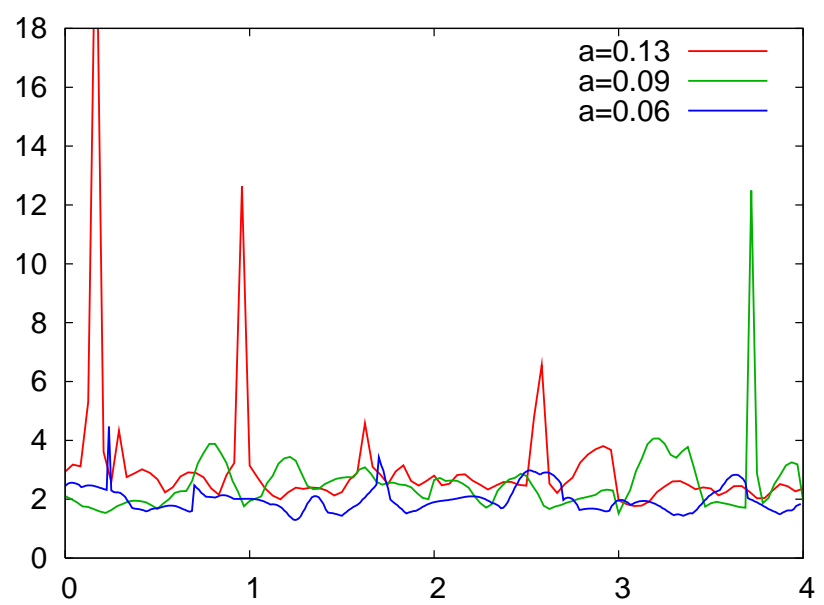

Figure 2: Time history of maximum value of the fermion force rescaled by the inverse time step for exploratory runs with three different lattice spacings. See Ref. [卂] for run parameters.

\begin{tabular}{|l|l|l|l|l|l|l|}
\hline$\beta$ & $a m_{l}$ & $a m_{s}$ & $a m_{c}$ & size & $a(\mathrm{fm})$ & $\mathrm{acpt}$ \\
\hline 5.80 & 0.0130 & 0.0650 & 0.838 & $16^{3} \times 48$ & 0.15 & 0.72 \\
6.00 & 0.0102 & 0.0509 & 0.635 & $20^{3} \times 64$ & 0.12 & 0.68 \\
6.30 & 0.0076 & 0.0380 & 0.445 & $28^{3} \times 96$ & 0.09 & 0.78 \\
6.65 & 0.0054 & 0.0270 & 0.313 & $48^{3} \times 144$ & 0.06 & 0.70 \\
\hline
\end{tabular}

Table 1: Parameters for tuning runs: coupling, dynamical quark masses in lattice units, lattice dimensions, approximate lattice spacing, and acceptance rate.

\begin{tabular}{|l|l|l|l|l|l|l|}
\hline$\beta$ & $a m_{l}$ & $a m_{s}$ & $a m_{c}$ & size & $a(\mathrm{fm})$ & $\mathrm{acpt}$ \\
\hline 6.00 & 0.0102 & 0.0509 & 0.635 & $24^{3} \times 64$ & 0.12 & 0.74 \\
6.30 & 0.0074 & 0.0370 & 0.440 & $32^{3} \times 96$ & 0.09 & 0.77 \\
\hline
\end{tabular}

Table 2: Same as for Table 1, except for production runs.

the asqtad action [2] and we compare results for a number of quantities. We find that the pion taste splittings are reduced by a factor of 2.5 to 3 , and that results with the HISQ action are comparable to those using the aqstad action at a lattice spacing of about 2/3 that used for the HISQ action.

\section{Initial results}

We present some initial results for the taste splittings of the pion, the spectrum of particles with light quarks, the $\eta_{c}$ dispersion relation and the topological susceptibility. In most cases, the results are compared with prior work on asqtad configurations and it is found that the results with the HISQ action look closer to the continuum limit. (We do not have results for the $\eta_{c}$ dispersion relation with asqtad quarks.) 


\subsection{Pion taste splittings}

It is well known that for staggered quarks, taste symmetry breaking is an important lattice artifact. A prime motivation for HISQ quarks is to improve the taste symmetry. In Fig. 3, we compare the pion taste splittings for the aqstad [4] and HISQ actions. We see that for $a \approx 0.12$ $\mathrm{fm}$, the taste splittings for the HISQ action are somewhat smaller than for asqtad with $a \approx 0.09 \mathrm{fm}$. Similarly, results using the HISQ action with $a \approx 0.09 \mathrm{fm}$ are similar to results using the asqtad action with $a \approx 0.06 \mathrm{fm}$. The vertical line to the left of this semi-log plot marked $\times 3$ is a ruler showing a factor of 3 . We see that using the HISQ action reduces the taste splitting by a factor of 2.5 to 3 compared with asqtad.

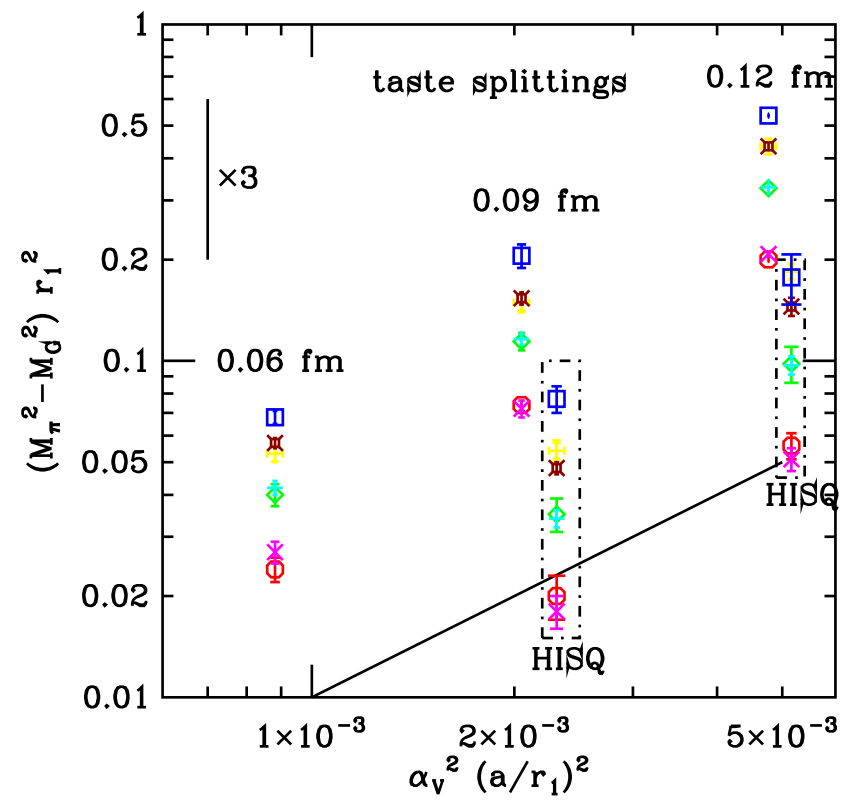

Figure 3: Calculation of taste splitting using the asqtad and HISQ actions. We show $\left(M_{\pi}^{2}-M_{G}^{2}\right) r_{1}^{2} v s$. $\alpha_{V}^{2}\left(a / r_{1}\right)^{2}$, where $M_{G}$ is the Goldstone taste state. The diagonal line on this log-log plot shows the slope of the expected dependence of the taste splitting.

\subsection{Spectrum of other light hadrons}

We also have some preliminary results for the masses of $\rho$, nucleon and $\Omega^{-}$. In each case, we find that using the HISQ action results in masses with smaller lattice spacing dependence, and the results are comparable to asqtad results at smaller lattice spacings. (The poster presented at the conference did not show results for $\rho$ due to lack of space.) In Figs. 4 and 5 , we show results for the $\rho$, nucleon and $\Omega^{-}$. The improvement for the $\rho$ seems quite substantial. For the nucleon, we find that the HISQ action result for $a \approx 0.15 \mathrm{fm}$ is quite a bit below the asqtad result with $a \approx 0.12 \mathrm{fm}$. The HISQ result for $a \approx 0.09 \mathrm{fm}$ appears comparable to that for the asqtad action for $a \approx 0.06 \mathrm{fm}$. The magenta curve represents a chiral perturbation theory fit to the asqtad results. For the $\Omega^{-}$we also see a substantial improvement of HISQ action results over asqtad action results with $a \approx 0.12$ and $0.09 \mathrm{fm}$. 


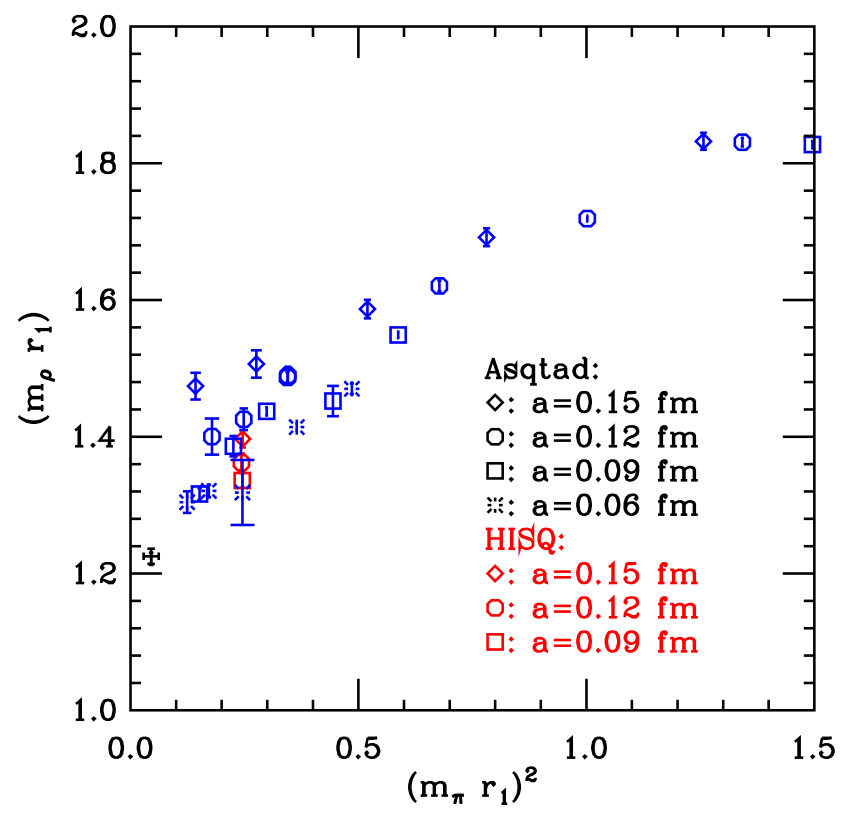

Figure 4: Rho mass in units of $r_{1} v s$. the pion mass squared.
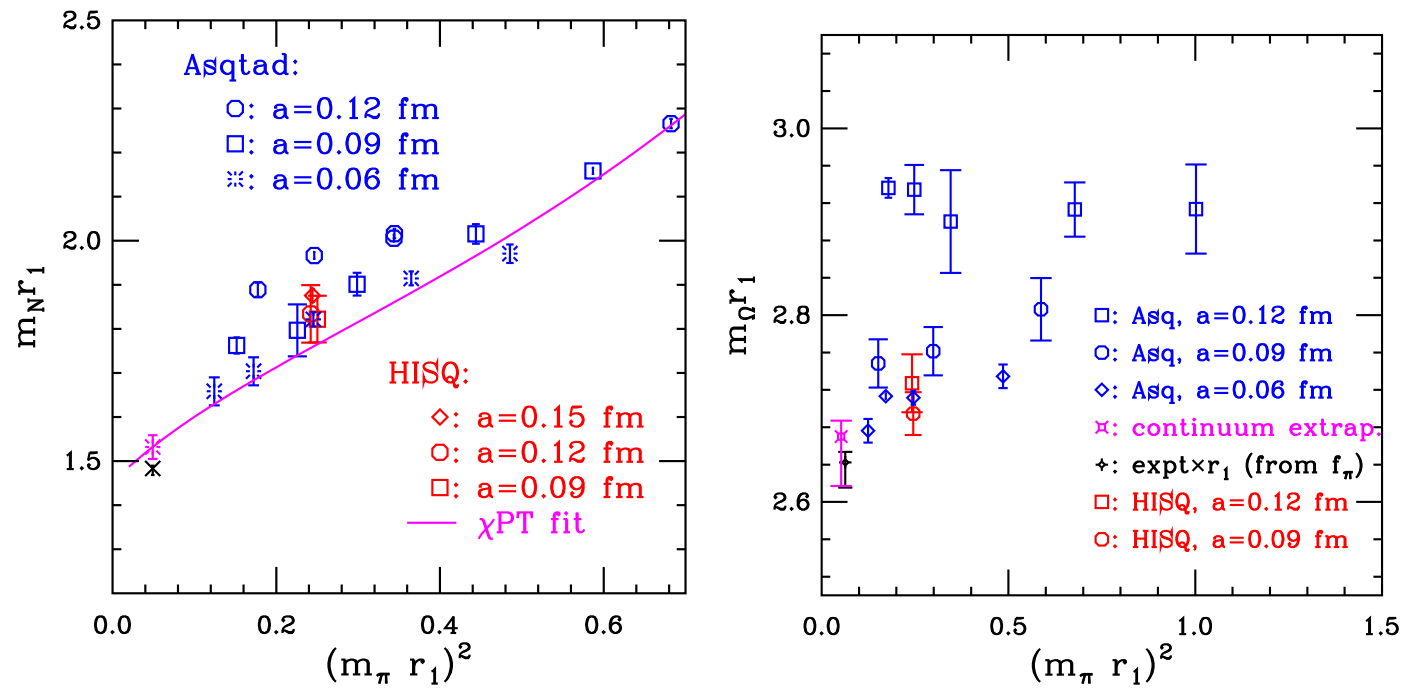

Figure 5: Nucleon mass (left) and $\Omega$ mass (right) in units of $r_{1} v s$. the pion mass squared.

\subsection{HISQ action and the charm quark}

The HPQCD/UKQCD collaboration has developed a procedure for tuning the coefficient of the Naik term to reduce discretization errors up to $\mathscr{O}\left(\left(a m_{c}\right)^{4}\right)$. At tree level, the correction to the Naik term expressed in terms of the bare charm quark mass is [3]

$$
\varepsilon=-\frac{27}{40}\left(a m_{c}\right)^{2}+\frac{327}{1120}\left(a m_{c}\right)^{4}-\frac{15607}{268800}\left(a m_{c}\right)^{6}-\frac{73697}{3942400}\left(a m_{c}\right)^{8} .
$$

Note that this formula differs from Eq. (24) in Ref. [3] because it is expressed in terms of the bare 
mass appearing in the action. In Fig. 6, we show the dispersion relation for the $\eta_{c}$ meson. For the three lattice spacings displayed, $-0.355 \leq \varepsilon \leq-0.123$. For $a \approx 0.06$ and $m_{l}=0.2 m_{s}, \varepsilon \approx-0.063$. We see that for a lattice spacing as coarse as $0.15 \mathrm{fm}$, the deviation from the continuum dispersion relation is as large as $7 \%$; however, when the lattice spacing is reduced to $0.09 \mathrm{fm}$ there is less than a $2 \%$ deviation from the continuum for the range of momentum shown here.

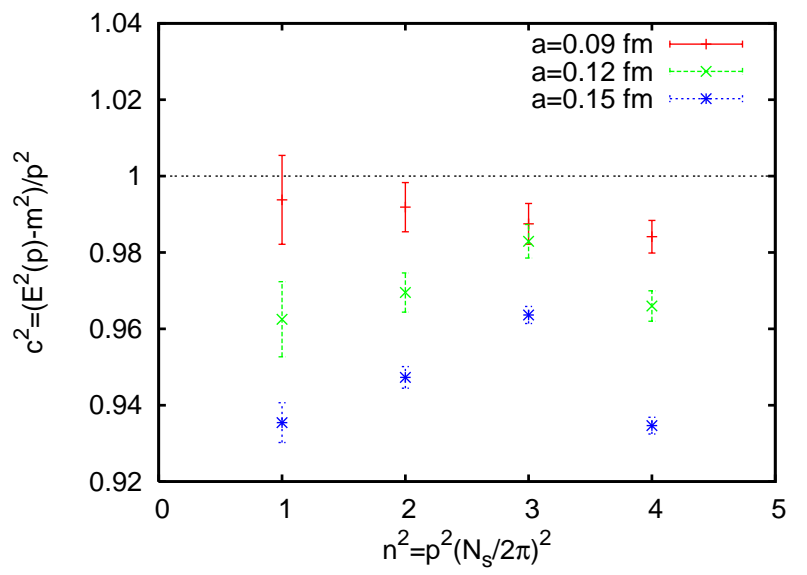

Figure 6: Speed of light from the $\eta_{c}$ dispersion relation $v s$. lattice momentum squared.

\subsection{Topological Susceptibility}

In Fig. 7, we plot the topological susceptibility for both asqtad and HISQ action simulations. We find that the HISQ action point for $a \approx 0.12 \mathrm{fm}$ falls close to the asqtad action curve for $a \approx 0.09$ fm. Also note that the HISQ action point lies far to the left of the corresponding asqtad action $a \approx 0.12$ point (the third green square from the left) since the HISQ action taste symmetry is so much better than for the asqtad action, and the horizontal axis involves the taste singlet pion mass as shown by Billeter, DeTar and Osborn [10]. The black points and curve are from a continuum extrapolation of the fits to the results for the three asqtad lattice spacings.

\section{Outlook}

Based on the encouraging results of our preliminary scaling study with $m_{l}=0.2 m_{s}$, the MILC collaboration is planning to generate additional ensembles with $m_{l}=0.1 m_{s}$ and $0.04 m_{s}$ for lattice spacings between 0.06 and $0.15 \mathrm{fm}$. These ensembles will allow us to study both the chiral and continuum limits. We expect to be able to study a wide variety of physics with these ensembles, for example, light pseudoscalar masses and decay constants, light quark spectroscopy, topology, spectroscopy of particles with a heavy quark, heavy-light meson decay constants and semileptonic decays, and quark masses.

\section{Acknowledgements}

This work was supported by the U.S. Department of Energy and the National Science Foundation. Computations were done on USQCD resources and at NICS and TACC. 


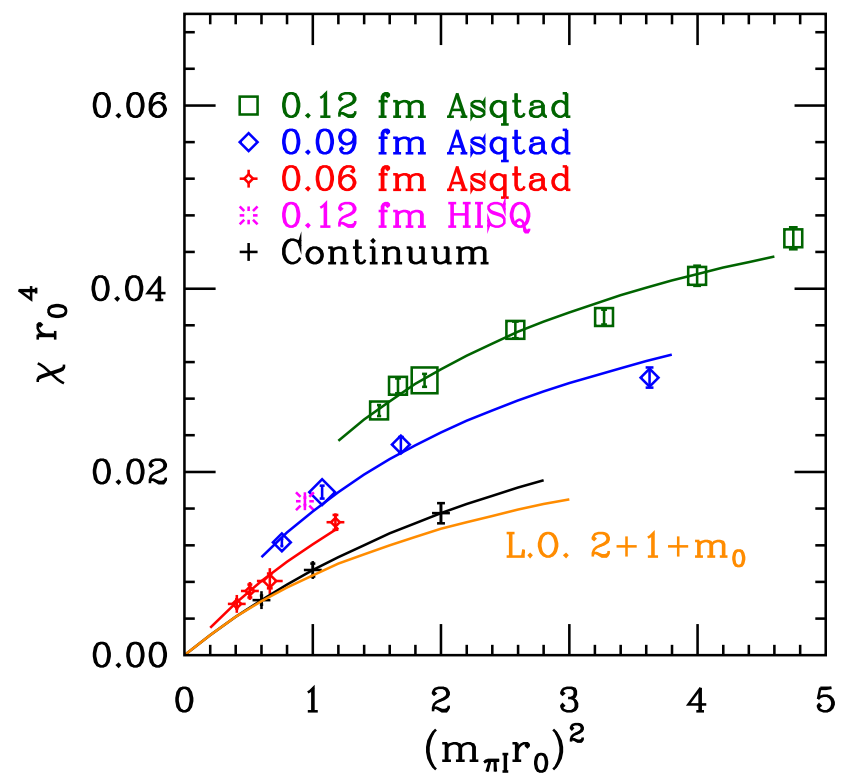

Figure 7: Topological susceptibility $v s$. squared mass of the taste singlet pion. The symbol size has been increased for the points coming from the asqtad action that correspond to $m_{l}=0.2 m_{s}$ so that they can more easily be compared with the HISQ action point. These points are either the second or third point from the left, depending on the lattice spacing.

\section{References}

[1] The MILC collaboration: C. Bernard et al., Nucl. Phys. (Proc. Suppl.), 60A (1998) 297; Phys. Rev. D 58 (1998) 014503; G.P. Lepage, Nucl. Phys. (Proc. Suppl.), 60A (1998) 267; Phys. Rev. D 59 (1999) 074501; K. Orginos and D. Toussaint (MILC), Nucl. Phys. (Proc. Suppl.), 73 (1999) 909; Phys. Rev. D 59 (1999) 014501; K. Orginos, D. Toussaint and R.L. Sugar (MILC), Phys. Rev. D 60 (1999) 054503; The MILC collaboration: C. Bernard et al., Phys. Rev. D 61 (2000) 111502.

[2] The MILC collaboration: A. Bazavov et al., arXiv:0903.3598 [hep-lat], to appear in Reviews of Modern Physics.

[3] E. Follana et al. [HPQCD collaboration and UKQCD collaboration], Phys. Rev. D 75 (2007) 054502 [arXiv:hep-lat/0610092].

[4] The MILC collaboration: A. Bazavov et al., PoS (LATTICE 2008) 033 [arXiv:0903.0874 [hep-lat]].

[5] W. Kamleh, D. B. Leinweber and A. G. Williams, Phys. Rev. D 70 (2004) 014502 [arXiv:hep-lat/0403019].

[6] K. Y. Wong and R. M. Woloshyn, PoS (LAT2007) 047 [arXiv:0710.0737 [hep-lat]].

[7] C. Morningstar and M. J. Peardon, Phys. Rev. D 69 (2004) 054501 [arXiv:hep-lat/0311018].

[8] A. Hasenfratz, R. Hoffmann and S. Schaefer, JHEP 0705 (2007) 029 [arXiv:hep-lat/0702028].

[9] M. A. Clark and A. D. Kennedy, Phys. Rev. Lett. 98 (2007) 051601, [arXiv:hep-lat/0608015]; Phys. Rev. D 75 (2007) 011502 [arXiv:hep-lat/0610047].

[10] B. Billeter, C. E. Detar and J. Osborn, Phys. Rev. D 70 (2004) 077502 [arXiv:hep-lat/0406032]. 